Louisiana State University

LSU Digital Commons

Faculty Publications

Department of Mathematics

$7-1-2020$

\title{
Sampled-Data Estimator for Nonlinear Systems with Arbitrarily Fast Rate of Convergence
}

Frederic Mazenc

Universite Paris-Saclay

Michael Malisoff

Louisiana State University

Silviu Iulian Niculescu

Universite Paris-Saclay

Follow this and additional works at: https://digitalcommons.Isu.edu/mathematics_pubs

\section{Recommended Citation}

Mazenc, F., Malisoff, M., \& Niculescu, S. (2020). Sampled-Data Estimator for Nonlinear Systems with Arbitrarily Fast Rate of Convergence. Proceedings of the American Control Conference, 2020-July, 1685-1689. https://doi.org/10.23919/ACC45564.2020.9147808

This Conference Proceeding is brought to you for free and open access by the Department of Mathematics at LSU Digital Commons. It has been accepted for inclusion in Faculty Publications by an authorized administrator of LSU Digital Commons. For more information, please contact ir@lsu.edu. 
archives-ouvertes

\title{
Sampled-Data Estimator for Nonlinear Systems with Arbitrarily Fast Rate of Convergence
}

\author{
Frederic Mazenc, Michael Malisoff, Silviu-Iulian Niculescu
}

\section{To cite this version:}

Frederic Mazenc, Michael Malisoff, Silviu-Iulian Niculescu. Sampled-Data Estimator for Nonlinear Systems with Arbitrarily Fast Rate of Convergence. ACC 2020 - American Control Conference, Jul 2020, Denver, France. pp.1685-1689, 10.23919/acc45564.2020.9147808 . hal-03103831

HAL Id: hal-03103831

https://hal-centralesupelec.archives-ouvertes.fr/hal-03103831

Submitted on 18 Jan 2021

HAL is a multi-disciplinary open access archive for the deposit and dissemination of scientific research documents, whether they are published or not. The documents may come from teaching and research institutions in France or abroad, or from public or private research centers.
L'archive ouverte pluridisciplinaire HAL, est destinée au dépôt et à la diffusion de documents scientifiques de niveau recherche, publiés ou non, émanant des établissements d'enseignement et de recherche français ou étrangers, des laboratoires publics ou privés. 


\section{Sampled-Data Estimator for Nonlinear Systems with Arbitrarily Fast Rate of Convergence}

\author{
Frederic Mazenc
}

\author{
Michael Malisoff
}

\author{
Silviu-Iulian Niculescu
}

\begin{abstract}
We study continuous-time nonlinear systems with discrete measurements. We provide an estimate of the state variable that converges with a rate of convergence that can be made arbitrarily large by reducing the size of the largest sampling interval. Our proof of the convergence result is based on a recently developed trajectory based approach.
\end{abstract}

Index Terms-Estimation, nonlinear systems, delay

\section{INTRODUCTION}

The design of estimators plays an important role in current research in control systems; see for instance [3], [4], [5], [6], and [15]. One important consideration for observer design is the speed at which the estimation error converges to 0 . For instance, [14] provided estimation results for a family of continuous-time systems which includes those of the type

$$
\left\{\begin{array}{l}
\dot{x}(t)=A x(t)+\mu(y(t), u(t)) \\
y(t)=C x(t)
\end{array}\right.
$$

where $A$ and $C$ are constant matrices, $x$ is the state, $y$ is the output, $\mu$ is a nonlinear function, and $u$ is an input which can be a control. In fact, [14] proposed a finite time observer. Its expression incorporates delays and no dynamic extension is used. The instant of convergence is arbitrarily small and can be chosen by the user, which is clearly an advantage over traditional asymptotically converging observers. The design in [14] is only applicable when the continuous output measurement $y(t)$ is available. This is a limitation of the result because, in many engineering applications, only discrete output measurements of the form

$$
y(t)=C x\left(t_{i}\right) \text { for all } t \in\left[t_{i}, t_{i+1}\right) \text { and } i \geq 0
$$

are available, where $t_{i}$ is an increasing sequence of nonnegative values. Then the observer of [14] cannot be constructed.

In the present work, our objective is to overcome this obstacle by proposing an adaptation of the technique of [14] to the case where the output is discrete and where the lengths $t_{i+1}-t_{i}$ of the sampling intervals are not required to be constant. This extension borrows key ideas from the celebrated paper [8], which, for nonlinear systems with discrete measurements, proposed a redesign of asymptotic observers that are available for systems with continuous measurements. The main ingredient in [8] is a dynamic extension which in

Malisoff is with the Department of Mathematics, Louisiana State University, Baton Rouge, LA 70803-4918, USA, malisoff@lsu.edu.

Mazenc and Niculescu are with EPI DISCO INRIA-Saclay, Laboratoire des Signaux et Systèmes (L2S, UMR CNRS 8506), CNRS, CentraleSupélec, Université Paris-Sud, 3 rue Joliot Curie, 91192, Gifsur-Yvette, France, frederic.mazenc@12s.centralesupelec.fr and Silviu.NICULESCU@12s.centralesupelec.fr.

Malisoff was supported by NSF Grant 1711299. some sense predicts the behavior of the measured variables between two measurements. We show that the key idea of the observer design of [8] used in combination with the approach of [14] produces an estimate of the state variable which converges with a rate of convergence that can be made arbitrarily large by reducing the size of the largest sampling interval. We study systems of the form

$$
\left\{\begin{array}{l}
\dot{x}(t)=A x(t)+\varphi(C x(t))+\kappa(u(t)) \\
y(t)=C x\left(t_{i}\right) \text { for all } t \in\left[t_{i}, t_{i+1}\right) \text { and } i \geq 0
\end{array}\right.
$$

with the state $x$ valued in $\mathbb{R}^{n}$ and the output $y$ valued in $\mathbb{R}$, where $u$ is an input, $C \in \mathbb{R}^{1 \times n}$ is a nonzero constant matrix, $\varphi$ and $\kappa$ are nonlinear functions, $A \in \mathbb{R}^{n \times n}$ is a constant matrix, and $t_{i}$ is an increasing sequence with $t_{0}=0$ and $\lim _{i \rightarrow+\infty} t_{i}=+\infty$. We allow nonperiodic sampling. We build a continuous-discrete observer which unlike the one of [14], will not converge in finite time but will converge with a rate of convergence of the form $c_{1} \ln \left(c_{2} \sup _{i}\left\{t_{i+1}-t_{i}\right\}\right)$ for suitable constants $c_{1}<0$ and $c_{2}>0$, which can be made arbitrarily large by sampling frequently enough. We prove our result through a recent trajectory based approach from [1], [12], and [13]. Since the observer of [14] relies on a formula with delays, one cannot directly apply [8] to solve the problem we study. The observer from [14] exhibited good performance that may be beyond the scope of high gain or other earlier observer designs, and we believe that the analog that we present here provides similar advantages. We leave the comparison of the performance of our observer design with the performance of other methods for future papers.

The notation will be simplified whenever no confusion can arise from the context. The Euclidean norm is denoted by $|\cdot|$. We let $I$ denote the identity matrix of any dimension. Given any constant $T>0$, we let $C_{\text {in }}$ denote the set of all continuous functions $\phi:[-T, 0] \rightarrow \mathbb{R}^{a}$. We define $\Xi_{t} \in C_{\text {in }}$ by $\Xi_{t}(m)=\Xi(t+m)$ for all choices of $\Xi, m \leq 0$, and $t \geq 0$ such that $t+m$ is in the domain of $\Xi$.

\section{MAIN RESUlT}

\section{A. Assumptions}

Our main assumptions on (3) are as follows:

Assumption 1: The pair $(A, C)$ is observable.

Assumption 2: There are constants $\bar{T}>0$ and $\underline{T}>0$ such that for all integers $i \geq 0$, the inequalities

$$
\underline{T} \leq t_{i+1}-t_{i} \leq \bar{T}
$$

are satisfied and $t_{0}=0$.

Assumption 3: The function $\kappa$ is continuous. Also, $\varphi$ is globally Lipschitz. 
By Assumption 1, we can find a constant $\tau>0$ (which can be chosen arbitrarily small) such that the matrix

$$
\Omega(\tau)=\left(\begin{array}{c}
C \\
C e^{-A \tau} \\
\vdots \\
C e^{-(n-1) A \tau}
\end{array}\right)
$$

is invertible. This can be done by choosing $\tau>0$ such that $\left(-A^{\top}, C^{\top}\right)$ is $\tau$-sampled controllable, using [16, Theorem 4 and Lemma 3.4.1] and the fact that the observability of $(A, C)$ implies that $\left(-A^{\top}, C^{\top}\right)$ is controllable. We also fix a global Lipschitz constant $\bar{\varphi} \geq 0$ for $\varphi$, so $\left|\varphi\left(y_{1}\right)-\varphi\left(y_{2}\right)\right| \leq$ $\bar{\varphi}\left|y_{1}-y_{2}\right|$ holds for all $y_{1} \in \mathbb{R}$ and $y_{2} \in \mathbb{R}$. We set

$$
\Psi(\tau)=\Omega(\tau)^{-1}
$$

$$
\begin{aligned}
& \lambda(\bar{T}, \tau)= \\
& \mathcal{F}(\tau) \sqrt{n}\left[1+|C|^{2} \bar{\varphi} e^{|A|(n-1) \tau}(\bar{T}+(n-1) \tau)\right]+|C| \bar{\varphi}, \\
& \text { and } \mathcal{F}(\tau)=|C A \Psi(\tau)| .
\end{aligned}
$$

Our final assumption is then as follows:

Assumption 4: The inequality

$$
\bar{T} \lambda(\bar{T}, \tau)<1
$$

is satisfied, and $\lambda(\bar{T}, \tau)$ is positive.

Remark 1: For any $A, C, \varphi$, and $\kappa$ such that Assumptions 1-3 are satisfied, one can determine a constant $\bar{T}>0$ such that (9) holds. For instance, this inequality is satisfied with

$$
\bar{T}=\min \left\{\frac{3}{4}, \frac{1}{\lambda(0.75, \tau)+\epsilon_{0}}\right\},
$$

for any positive constant $\epsilon_{0}$.

\section{B. Estimator Design}

Let us introduce the continuous-discrete system

$$
\left\{\begin{aligned}
\dot{\omega}(t)= & C A \hat{x}(t)+C \varphi(\omega(t))+C \kappa(u(t)) \\
& \text { for all } t \in\left[t_{i}, t_{i+1}\right) \text { and } i \geq 0 \\
\omega\left(t_{i}\right)= & C x\left(t_{i}\right) \text { for all } i \geq 0 \\
\hat{x}(t)= & \Psi(\tau) \mathcal{U}_{1}\left(\omega_{t}\right)+\Psi(\tau) \mathcal{U}_{2}\left(\omega_{t}, u_{t}\right)
\end{aligned}\right.
$$

with $\hat{x}$ valued in $\mathbb{R}^{n}, \omega$ valued in $\mathbb{R}, \tau>0$ being a constant such that $\Omega(\tau)$ is invertible,

$$
\begin{gathered}
\mathcal{U}_{1}\left(\omega_{t}\right)=\left(\begin{array}{c}
\omega(t) \\
\omega(t-\tau) \\
\vdots \\
\omega(t-(n-1) \tau)
\end{array}\right) \text { and } \\
\mathcal{U}_{2}\left(\omega_{t}, u_{t}\right)=\left(\begin{array}{c}
0 \\
C e^{-\tau A} \Delta H_{1}(t) \\
\vdots \\
C e^{-(n-1) \tau A} \Delta H_{n-1}(t)
\end{array}\right)
\end{gathered}
$$

and where $\Delta H_{j}(t)=H_{j}(t)-e^{j A \tau} H_{j}(t-j \tau)$ and the $H_{j}$ 's solve

$$
\dot{H}_{j}(t)=A H_{j}(t)+\varphi(\omega(t))+\kappa(u(t))
$$

for $j=1, \ldots, n-1$ and any constant initial functions that are defined over $[-(n-1) \tau, 0]$. The $\omega$-subsystem of this observer is inspired by the one from [8] and $\hat{x}$ is reminiscent of the observer of [14]. Although we can apply variation of parameters on the intervals $[t-j \tau, t]$ to check that

$$
\begin{aligned}
& e^{-j A \tau}\left(H_{j}(t)-e^{j A \tau} H_{j}(t-j \tau)\right)= \\
& \int_{t-j \tau}^{t} e^{A(t-m-j \tau)}[\varphi(\omega(m))+\kappa(u(m))] \mathrm{d} m
\end{aligned}
$$

holds for all $t \geq(n-1) \tau$ and $j=1,2, \ldots, n-1$, we express (13) using solutions of (14) to obtain formulas for estimators that do not contain any integrations. Our main result is:

Theorem 1: Let (3) satisfy Assumptions 1-4. Then for all constant initial functions for (3) and (11), and with the choice $c_{*}=\sqrt{n}|\Psi(\tau)|\left(1+|C|(n-1) \bar{\varphi} \tau e^{(n-1)|A| \tau}\right)$, we have

$$
\begin{aligned}
& |\omega(t)-C x(t)| \leq \\
& \sup _{\ell \in[0, \bar{T}+(n-1) \tau]}|\omega(\ell)-C x(\ell)| e^{\frac{\ln (\bar{T} \lambda(\bar{T}, \tau))}{2(\bar{T}+(n-1) \tau)}(t-\bar{T}-(n-1) \tau)}
\end{aligned}
$$

for all $t \geq \bar{T}+(n-1) \tau$ and

$|x(t)-\hat{x}(t)| \leq$

$c_{*} \sup _{\ell \in[0, \bar{T}+(n-1) \tau]}|\omega(\ell)-C x(\ell)| e^{\frac{\ln (\bar{T} \lambda(\bar{T}, \tau))}{2(\bar{T}+2(n-1) \tau)}(t-\bar{T}-(n-1) \tau)}$

for all $t \geq \bar{T}+2(n-1) \tau$.

Remark 2: Our observer (11)-(14) entails resetting the $\omega$ values at each time $t_{i}$ when a new output value becomes available. Hence, at each time $t$, future sampling times can be uncertain. Theorem 1 provides the rate of convergence $r=c_{1} \ln \left(c_{2} \bar{T}\right)$ of $|x(t)-\hat{x}(t)|$ to 0 , where

$$
c_{1}=-\frac{1}{2(\bar{T}+2(n-1) \tau)}<0 \text { and } c_{2}=\lambda(\bar{T}, \tau)>0,
$$

and $r$ diverges to $\infty$ as $\bar{T} \rightarrow 0$. Thus, the continuous-time case, where an exact estimate of the state is obtained in finite time, can be seen as the limiting case as $\bar{T} \rightarrow 0$.

Remark 3: An upper bound for $|\omega(t)-C x(t)|$ over the interval $[0, \bar{T}+(n-1) \tau]$ depending on the initial conditions can be obtained. However since $[0, \bar{T}+(n-1) \tau]$ is in a sense arbitrary (because $\tau$ and $\bar{T}$ are chosen by the user), the behavior of the solutions over this interval has no significant interest from a practical point of view.

Remark 4: Several observers in the literature can be applied to (3), notably those of [2], [7], and [11]. However, we believe that they either use high gain or do not achieve the arbitrarily fast convergence property from Theorem 1 .

\section{Proof OF Theorem 1}

To simplify the proof, we consider the case where the function $\kappa$ is not present. The extension to the general case is straightforward, by introducing $\kappa(u(m))$ in the integrals in (19)-(23).

First note that Assumptions 2-3 ensure that the system consisting of (3) and (11) is forward complete and that the socalled chattering phenomenon does not occur. By integrating (3) over any interval $[s, t]$ with $0 \leq s \leq t$, we obtain

$$
x(t)=e^{A(t-s)} x(s)+\int_{s}^{t} e^{A(t-m)} \varphi(C x(m)) \mathrm{d} m .
$$


Thus, for all $p \in\{0, \ldots, n-1\}$, we have

$$
\begin{aligned}
C e^{-p A \tau} x(t)= & C x(t-p \tau) \\
& +C \int_{t-p \tau}^{t} e^{A(t-m-p \tau)} \varphi(C x(m)) \mathrm{d} m
\end{aligned}
$$

for all $t \geq p \tau$. Setting

$$
\Delta_{1}\left(x_{t}\right)=\Psi(\tau)\left(\begin{array}{c}
C x(t) \\
C x(t-\tau) \\
\vdots \\
C x(t-(n-1) \tau)
\end{array}\right) \text { and }
$$

$$
\begin{aligned}
& \Delta_{2}\left(x_{t}\right)= \\
& \Psi(\tau)\left(\begin{array}{c}
0 \\
C \int_{t-\tau}^{t} e^{A(t-m-\tau)} \varphi(C x(m)) \mathrm{d} m \\
\vdots \\
C \int_{t-(n-1) \tau}^{t} e^{A(t-m-(n-1) \tau)} \varphi(C x(m)) \mathrm{d} m
\end{array}\right),
\end{aligned}
$$

we easily deduce from (20) that

$$
x(t)=\Delta_{1}\left(x_{t}\right)+\Delta_{2}\left(x_{t}\right)
$$

for all $t \geq(n-1) \tau$.

Let us introduce the variables

$$
e_{\omega}(t)=\omega(t)-C x(t) \text { and } e_{x}(t)=\hat{x}(t)-x(t)
$$

and let $\underline{i}$ be the integer such that $t_{i-1}<(n-1) \tau$ and $t_{i} \geq$ $(n-1) \tau$. Then in terms of the functions

$$
D_{3, i}\left(x_{t}, \omega_{t}\right)=\omega(t-i \tau)-C x(t-i \tau)
$$

for $i=0, \ldots, n-1$ and

$$
\begin{aligned}
& D_{4, i}\left(x_{t}, \omega_{t}\right)= \\
& C \int_{t-i \tau}^{t} e^{A(t-m-i \tau)}[\varphi(\omega(m))-\varphi(C x(m))] \mathrm{d} m
\end{aligned}
$$

for $i=1,2, \ldots, n-1$, one can use (11)-(13) and (15) to prove that

$$
\left\{\begin{aligned}
\dot{e}_{\omega}(t)= & C A \hat{x}(t)+C \varphi(\omega(t))-C A x(t) \\
& -C \varphi(C x(t)) \text { for all } t \in\left[t_{i}, t_{i+1}\right) \\
e_{\omega}\left(t_{i}\right)= & 0 \\
e_{x}(t)= & \Psi(\tau)\left(\begin{array}{c}
D_{3,0}\left(x_{t}, \omega_{t}\right) \\
D_{3,1}\left(x_{t}, \omega_{t}\right) \\
\vdots \\
D_{3, n-1}\left(x_{t}, \omega_{t}\right) \\
0 \\
\end{array}\right. \\
& +\Psi(\tau)\left(\begin{array}{c}
D_{4,1}\left(x_{t}, \omega_{t}\right) \\
\vdots \\
D_{4, n-1}\left(x_{t}, \omega_{t}\right)
\end{array}\right)
\end{aligned}\right.
$$

for all $i \in \mathbb{N}$ with $i \geq \underline{i}$ or, equivalently,

$$
\left\{\begin{aligned}
\dot{e}_{\omega}(t)= & C A e_{x}(t)+C[\varphi(\omega(t))-\varphi(C x(t))] \\
& \text { for all } t \in\left[t_{i}, t_{i+1}\right) \\
e_{\omega}\left(t_{i}\right)= & 0 \\
e_{x}(t)= & \Psi(\tau) \mathcal{U}_{1}\left(e_{\omega, t}\right)+\Psi(\tau)\left(\begin{array}{c}
0 \\
D_{4,1}\left(x_{t}, \omega_{t}\right) \\
\vdots \\
D_{4, n-1}\left(x_{t}, \omega_{t}\right)
\end{array}\right)
\end{aligned}\right.
$$

for all $i \in \mathbb{N}$ with $i \geq \underline{i}$ and with $\mathcal{U}_{1}$ as defined in (12) (with $\omega_{t}$ replaced by $e_{\omega, t}$ in the $\mathcal{U}_{1}$ formula in (12)). Hence,

$$
\left\{\begin{aligned}
\dot{e}_{\omega}(t)= & C A \Psi(\tau) \mathcal{U}_{1}\left(e_{\omega, t}\right) \\
& +C A \Psi(\tau)\left(\begin{array}{c}
0 \\
D_{4,1}\left(x_{t}, \omega_{t}\right) \\
\vdots \\
D_{4, n-1}\left(x_{t}, \omega_{t}\right)
\end{array}\right) \\
& +C[\varphi(\omega(t))-\varphi(C x(t))] \\
& \text { for all } t \in\left[t_{i}, t_{i+1}\right) \\
e_{\omega}\left(t_{i}\right)= & 0
\end{aligned}\right.
$$

for all $i \in \mathbb{N}$ with $i \geq i$.

We deduce by integrating (29) over the interval $\left[t_{i}, t\right)$ that

$$
\begin{aligned}
& e_{\omega}(t)=C \int_{t_{i}}^{t}[\varphi(\omega(\ell))-\varphi(C x(\ell))] \mathrm{d} \ell \\
& +C A \Psi(\tau) \mathcal{U}_{3}\left(t, x_{t}, \omega_{t}\right)+C A \Psi(\tau) \mathcal{U}_{4}\left(t, x_{t}, \omega_{t}\right)
\end{aligned}
$$

for all $t \geq 0$ and $i \in \mathbb{N}$ such that $t \in\left[t_{i}, t_{i+1}\right)$, where

$$
\mathcal{U}_{3}\left(t, x_{t}, \omega_{t}\right)=\left(\begin{array}{c}
\int_{t_{i}}^{t} e_{\omega}(\ell) \mathrm{d} \ell \\
\int_{t_{i}}^{t} e_{\omega}(\ell-\tau) \mathrm{d} \ell \\
\vdots \\
\int_{t_{i}}^{t} e_{\omega}(\ell-(n-1) \tau) \mathrm{d} \ell
\end{array}\right)
$$

and

$$
\mathcal{U}_{4}\left(t, x_{t}, \omega_{t}\right)=\left(\begin{array}{c}
0 \\
C \mathcal{U}_{4,1}\left(t, x_{t}, \omega_{t}\right) \\
\vdots \\
C \mathcal{U}_{4, n-1}\left(t, x_{t}, \omega_{t}\right)
\end{array}\right)
$$

and

$$
\begin{aligned}
& \mathcal{U}_{4, i}\left(t, x_{t}, \omega_{t}\right)= \\
& \int_{t_{i}}^{t} \int_{\ell-i \tau}^{\ell} e^{A(\ell-m-i \tau)}[\varphi(\omega(m))-\varphi(C x(m))] \mathrm{d} m \mathrm{~d} \ell
\end{aligned}
$$

for $i \geq \underline{i}$.

The equality (30) implies that

$$
\begin{aligned}
& \left|e_{\omega}(t)\right| \leq \mathcal{F}(\tau) \sqrt{\sum_{l=0}^{n-1}\left|\int_{t_{i}}^{t} e_{\omega}(\ell-l \tau) \mathrm{d} \ell\right|^{2}} \\
& +\mathcal{F}(\tau)|C| \sqrt{\sum_{l=1}^{n-1}\left|\mathcal{U}_{4, l}\left(t, x_{t}, \omega_{t}\right)\right|^{2}} \\
& +|C| \int_{t_{i}}^{t}|\varphi(\omega(\ell))-\varphi(C x(\ell))| \mathrm{d} \ell
\end{aligned}
$$

where $\mathcal{F}$ is from (8). From Assumption 3, we deduce that

$$
\begin{aligned}
& \left|e_{\omega}(t)\right| \leq \mathcal{F}(\tau)\left\{\sqrt{\sum_{l=0}^{n-1}\left|\int_{t_{i}-l \tau}^{t-l \tau} e_{\omega}(\ell) \mathrm{d} \ell\right|^{2}}\right. \\
& \left.+|C|^{2} \bar{\varphi} \sqrt{\sum_{l=1}^{n-1}\left(\int_{t_{i}}^{t} \int_{\ell-l \tau}^{\ell}\left|e^{A(\ell-m-l \tau)}\right|\left|e_{\omega}(m)\right| \mathrm{d} m \mathrm{~d} \ell\right)^{2}}\right\} \\
& +|C| \bar{\varphi} \int_{t_{i}}^{t}\left|e_{\omega}(\ell)\right| \mathrm{d} \ell .
\end{aligned}
$$


By observing that

$$
\begin{aligned}
& \int_{t_{i}}^{t} \int_{\ell-l \tau}^{\ell}\left|e^{A(\ell-m-l \tau)}\right|\left|e_{\omega}(m)\right| \mathrm{d} m \mathrm{~d} \ell \\
& \leq e^{|A|(n-1) \tau} \int_{t_{i}}^{t} \int_{\ell-l \tau}^{\ell}\left|e_{\omega}(m)\right| \mathrm{d} m \mathrm{~d} \ell \\
& \leq e^{|A|(n-1) \tau}\left(t-t_{i}\right) \int_{t_{i}-(n-1) \tau}^{t}\left|e_{\omega}(m)\right| \mathrm{d} m \\
& \leq e^{|A|(n-1) \tau} \bar{T} \int_{t_{i}-(n-1) \tau}^{t}\left|e_{\omega}(m)\right| \mathrm{d} m
\end{aligned}
$$

and

$$
\begin{aligned}
& \left|\int_{t_{i}-l \tau}^{t-l \tau} e_{\omega}(\ell) \mathrm{d} \ell\right|^{2} \leq\left(t-t_{i}\right) \int_{t_{i}-l \tau}^{t-l \tau}\left|e_{\omega}(\ell)\right|^{2} \mathrm{~d} \ell \\
& \leq \bar{T} \int_{t_{i}-l \tau}^{t-l \tau}\left|e_{\omega}(\ell)\right|^{2} \mathrm{~d} \ell
\end{aligned}
$$

for all $l \in\{1, \ldots, n-1\}$, where (36) is a consequence of Jensen's inequality, we deduce that

$$
\begin{aligned}
\left|e_{\omega}(t)\right| \leq & \mathcal{F}(\tau) \sqrt{\bar{T}} \sqrt{\sum_{l=0}^{n-1} \int_{t_{i}-l \tau}^{t-l \tau}\left|e_{\omega}(\ell)\right|^{2} \mathrm{~d} \ell} \\
& +\mathcal{G}(\tau) \bar{T} \int_{t_{i}-(n-1) \tau}^{t}\left|e_{\omega}(m)\right| \mathrm{d} m \\
& +|C| \bar{\varphi} \int_{t_{i}}^{t}\left|e_{\omega}(\ell)\right| \mathrm{d} \ell \\
\leq & \mathcal{F}(\tau) \sqrt{\bar{T} n\left(t-t_{i}\right)} \sup _{\ell \in\left[t_{i}-(n-1) \tau, t\right]}\left|e_{\omega}(\ell)\right| \\
& +\mathcal{L}(t) \sup _{\ell \in\left[t_{i}-(n-1) \tau, t\right]}\left|e_{\omega}(\ell)\right| \\
& +|C| \bar{\varphi}\left(t-t_{i}\right) \sup _{\ell \in\left[t_{i}, t\right]}\left|e_{\omega}(\ell)\right|
\end{aligned}
$$

with

$$
\mathcal{L}(t)=\mathcal{G}(\tau) \bar{T}\left[t-t_{i}+(n-1) \tau\right]
$$

and

$$
\mathcal{G}(\tau)=\mathcal{F}(\tau)|C|^{2} \bar{\varphi} e^{|A|(n-1) \tau} \sqrt{n} .
$$

Since $t \in\left[t_{i}, t_{i+1}\right)$, it follows that

$$
\begin{aligned}
& \left|e_{\omega}(t)\right| \leq \bar{T} \mathcal{F}(\tau) \sqrt{n} \sup _{\ell \in\left[t_{i}-(n-1) \tau, t\right]}\left|e_{\omega}(\ell)\right| \\
& +\mathcal{G}(\tau) \bar{T}(\bar{T}+(n-1) \tau) \sup _{\ell \in\left[t_{i}-(n-1) \tau, t\right]}\left|e_{\omega}(\ell)\right| \\
& +|C| \bar{\varphi} \bar{T} \sup _{\ell \in\left[t_{i}, t\right]}\left|e_{\omega}(\ell)\right| .
\end{aligned}
$$

Using the inequality

$$
\sup _{\ell \in\left[t_{i}, t\right]}\left|e_{\omega}(\ell)\right| \leq \sup _{\ell \in\left[t_{i}-(n-1) \tau, t\right]}\left|e_{\omega}(\ell)\right|,
$$

we obtain

$$
\left|e_{\omega}(t)\right| \leq \bar{T} \lambda(\bar{T}, \tau) \sup _{\ell \in[t-\bar{T}-(n-1) \tau, t]}\left|e_{\omega}(\ell)\right|,
$$

where $\lambda(\bar{T}, \tau)$ is the function defined in (7) for all $t \geq \bar{T}+$ $(n-1) \tau$. Then (9) in Assumption 4 and [12, Lemma 1] (which we also include in Appendix A below) applied to the function $z(t)=e_{\omega}(t+\bar{T}+(n-1) \tau)$ and the constant $T^{*}=2(\bar{T}+(n-1) \tau)$ ensure that

$$
\begin{aligned}
& \left|e_{\omega}(t)\right| \leq \\
& \sup _{\ell \in[0, \bar{T}+(n-1) \tau]}\left|e_{\omega}(\ell)\right| e^{\frac{\ln (\bar{T} \lambda(\bar{T}, \tau))}{2(\bar{T}+(n-1) \tau)}(t-\bar{T}-(n-1) \tau)}
\end{aligned}
$$

for all $t \geq \bar{T}+(n-1) \tau$. This concludes the proof of the first conclusion of the theorem. The second conclusion now follows from the formula for $e_{x}$ in (27).

\section{ILluStration}

\section{A. Studied System: Special Case of (3)}

We illustrate Theorem 1 in the particular case where

$$
\begin{gathered}
A=\left(\begin{array}{rr}
0 & 1 \\
-1 & 0
\end{array}\right), C=\left(\begin{array}{ll}
1 & 0
\end{array}\right), \\
\varphi(y)=\epsilon \sin (y)\left(\begin{array}{l}
1 \\
0
\end{array}\right), \text { and } \kappa(u)=\left(\begin{array}{c}
u^{2} \\
-u
\end{array}\right)
\end{gathered}
$$

for any constant $\epsilon \in \mathbb{R}$. Then Assumptions 1 and 3 are satisfied and $\bar{\varphi}=\epsilon$. Since

$$
e^{-A m}=\left(\begin{array}{cc}
\cos (m) & -\sin (m) \\
\sin (m) & \cos (m)
\end{array}\right)
$$

for all $m \in \mathbb{R}$, we deduce that

$$
\Omega(\tau)=\left(\begin{array}{c}
C \\
C e^{-A \tau}
\end{array}\right)=\left(\begin{array}{cc}
1 & 0 \\
\cos (\tau) & -\sin (\tau)
\end{array}\right)
$$

and for any $\tau \in\left(0, \frac{\pi}{2}\right]$, we have

$$
\Psi(\tau)=\left(\begin{array}{cc}
1 & 0 \\
\frac{\cos (\tau)}{\sin (\tau)} & -\frac{1}{\sin (\tau)}
\end{array}\right) .
$$

Thus, in terms of the notation from Theorem 1, we have

$$
\begin{aligned}
& \Psi\left(\frac{\pi}{2}\right)=\left(\begin{array}{cc}
1 & 0 \\
0 & -1
\end{array}\right), \mathcal{F}\left(\frac{\pi}{2}\right)=1, \\
& \text { and } \lambda\left(\bar{T}, \frac{\pi}{2}\right)=\sqrt{2}\left[1+\epsilon e^{\frac{\pi}{2}}\left(\bar{T}+\frac{\pi}{2}\right)\right]+\epsilon .
\end{aligned}
$$

With for instance $\epsilon=\frac{1}{6}$, we get

$$
\lambda\left(\bar{T}, \frac{\pi}{2}\right)=\sqrt{2}\left[1+\frac{e^{\frac{\pi}{2}}}{6}\left(\bar{T}+\frac{\pi}{2}\right)\right]+\frac{1}{6} .
$$

Then Assumption 4 is satisfied with

$$
\bar{T}=\frac{1}{\sqrt{2}\left[1+\frac{e^{\frac{\pi}{2}}}{6}\left(0.3+\frac{\pi}{2}\right)\right]+1} .
$$

It follows that with the choice $\tau=\pi / 2$, Theorem 1 applies to the system we consider and it provides the observer

$$
\left\{\begin{aligned}
\dot{\omega}(t)= & \hat{x}_{2}(t)+\frac{1}{6} \sin (\omega(t))+u^{2}(t) \\
& \text { for all } t \in\left[t_{i}, t_{i+1}\right) \text { and } i \geq 0 \\
\omega\left(t_{i}\right)= & x_{1}\left(t_{i}\right) \text { for all } i \geq 0 \\
\hat{x}(t)= & \left(\begin{array}{c}
\omega(t) \\
-\omega\left(t-\frac{\pi}{2}\right)
\end{array}\right)-\left(\begin{array}{c}
0 \\
\mathcal{R}_{a}(t)
\end{array}\right),
\end{aligned}\right.
$$


where

$$
\begin{aligned}
& \mathcal{R}_{a}(t)= \\
& \int_{t-\frac{\pi}{2}}^{t}\left(\cos \left(t-m-\frac{\pi}{2}\right)\left[\epsilon \sin (\omega(m))+u^{2}(m)\right]\right. \\
& \left.-\sin \left(t-m-\frac{\pi}{2}\right) u(m)\right) \mathrm{d} m .
\end{aligned}
$$

\section{B. Simulation}

In this part, we present numerical simulations, using Mathematica. We select the sampling instants $t_{i}=i j \bar{T}$ with $\bar{T}$ defined in (51) and the input $u(t)=\sin (t)$, with the choice $j=1$, then with $j=1 / 2$, and finally with $j=0.25$. We choose the initial conditions $x_{1}(0)=x_{2}(0)=1$ and $w(0)=0$. In Fig. 1, we plot the resulting observation error values of $\hat{x}_{2}(t)-x_{2}(t)$ for the three different choices of the sampling rate. Since the figure shows rapid convergence of the errors to 0 , with faster convergence as $j$ decreases, it helps illustrate our theorem in the special case of (44)-(45).

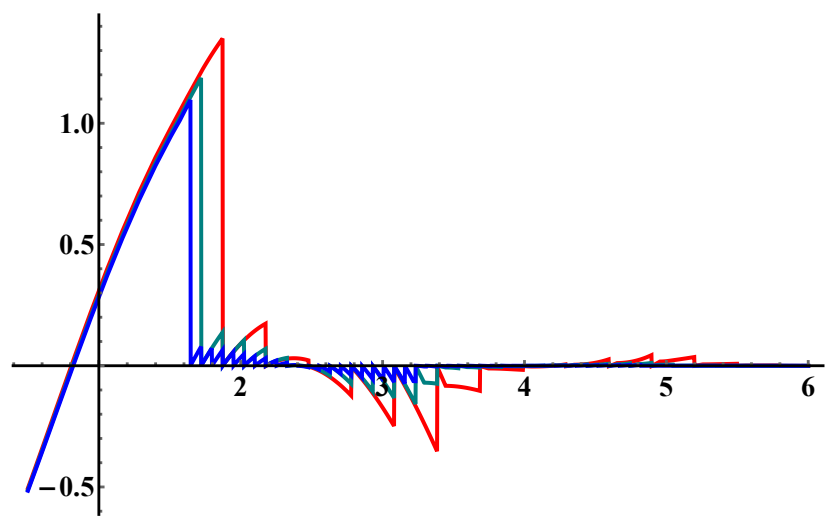

Fig. 1. Tracking Error $\hat{x}_{2}(t)-x_{2}(t)$ Converging to 0 over Time with Sampling Rates $\bar{T}$ (Red), $0.5 \bar{T}$ (Green), and $0.25 \bar{T}$ (Blue) with $\bar{T}$ as defined in (51).

\section{Conclusions}

We extended the observer design from [14] to cases where the measurements are discrete. A key novel feature was our use of a trajectory based approach, which enabled us to ensure arbitrarily fast convergence of the observation error to zero, provided the sampling in the output is frequent enough. Our trajectory based approach used a contractivity condition instead of a Lyapunov function, and the sampling rate is chosen so that the constant of contractivity $\rho$ satisfies the requirement $\rho \in(0,1)$. Many extensions can be expected. They pertain to time-varying systems, systems with delay in the measurements, robustness with respect to additive disturbances or the vector field of the system, local observer design of systems with general nonlinear terms, and multirate sampled-data observers in the spirit of what is proposed in [9] and [10] in the case where the dimension of the output is larger than 1 .

\section{Appendix A: Statement of [12, Lemma 1]}

We provide a statement of a special case of [12, Lemma 1], which we used at the end of our proof of Theorem 1 above:
Lemma A.1: Let $T^{*}>0$ be a constant. Let a piecewise continuous function $z:\left[-T^{*},+\infty\right) \rightarrow[0,+\infty)$ admit a sequence of real numbers $v_{i}$ and positive constants $\bar{v}_{a}$ and $\bar{v}_{b}$ such that $v_{0}=0, v_{i+i}-v_{i} \in\left[\bar{v}_{a}, \bar{v}_{b}\right]$ for all $i \geq 0, z$ is continuous on each interval $\left[v_{i}, v_{i+1}\right)$ for all $i \geq 0$, and $z\left(v_{i}^{-}\right)$exists and is finite for each $i \in \mathbb{N}$. Assume that there is a constant $\rho \in(0,1)$ such that

$$
z(t) \leq \rho \sup _{\ell \in\left[t-T^{*}, t\right]}|z(\ell)|
$$

holds for all $t \geq 0$. Then

$$
z(t) \leq \sup _{\ell \in\left[-T^{*}, 0\right]}|z(\ell)| e^{\frac{\ln (\rho)}{T^{*}} t}
$$

holds for all $t \geq 0$.

\section{REFERENCES}

[1] S. Ahmed, F. Mazenc, and H. Ozbay. Dynamic output feedback stabilization of switched linear systems with delay via a trajectory based approach. Automatica, 93:92-97, 2018.

[2] V. Andrieu and M. Nadri. Observer design for Lipschitz systems with discrete-time measurements. In Proceedings of the 49th IEEE Conference on Decision and Control, pages 6522-6527, Atlanta, GA, 2010.

[3] A. Borri, F. Cacace, A. De Gaetano, A. Germani, C. Manes, P. Palumbo, S. Panunzi, and P. Pepe. Luenberger-like observers for nonlinear time-delay systems with application to the artificial pancreas: the attainment of good performance. IEEE Control Systems Magazine, 37(4):33-49, 2017.

[4] C. Buccella, C. Cecati, H. Latafat, P. Pepe, and K. Razi. Observerbased control of LCDC/DC resonant converter using extended describing functions. IEEE Transactions on Power Electronics, 30(10):58815891, 2014.

[5] F. Cacace, A. Germani, and C. Manes. A chain observer for nonlinear systems with multiple time-varying measurement delays. SIAM Journal on Control and Optimization, 52(3):1862-1885, 2014.

[6] F. Cacace, A. Germani, and C. Manes. A new approach to design interval observers for linear systems. IEEE Transactions on Automatic Control, 60(6): 1665-1670, 2014.

[7] T. Dinh, V. Andrieu, M. Nadri, and U. Serres. Continuous-discrete time observer design for Lipschitz systems with sampled measurements. IEEE Transactions on Automatic Control, 60(3):787-792, 2015.

[8] I. Karafyllis and C. Kravaris. From continuous-time design to sampleddata design of observers. IEEE Transactions on Automatic Control, 54(9):2169-2174, 2009.

[9] C. Ling and C. Kravaris. Multi-rate sampled-data observer design for nonlinear systems with asynchronous and delayed measurements. In Proceedings of the American Control Conference, pages 1128-1133, Philadelphia, PA, 2019.

[10] C. Ling and C. Kravaris. Multi-rate sampled-data observer design based on a continuous-time design. IEEE Transactions on Automatic Control, 2019, to appear, preprint available at https://doiorg.libezp.lib.lsu.edu/10.1109/TAC.2019.2929295.

[11] F. Mazenc, V. Andrieu, and M. Malisoff. Design of continuous-discrete observers for time-varying nonlinear systems. Automatica, 57(7):135$144,2015$.

[12] F. Mazenc and M. Malisoff. Trajectory based approach for the stability analysis of nonlinear systems with time delays. IEEE Transactions on Automatic Control, 60(6):1716-1721, 2015.

[13] F. Mazenc, M. Malisoff, and S-I. Niculescu. Stability and control design for time-varying systems with time-varying delays using a trajectory based approach. SIAM Journal on Control and Optimization, 55(1):533-556, 2017.

[14] F. Mazenc, S-I. Niculescu, and N. Bekiaris-Liberis. Asymptotic stabilization of linear time-varying systems with input delays via delayed static output feedback. In Proceedings of the American Control Conference, pages 4591-4596, Chicago, IL, 2015.

[15] A. Parikh, T-H. Cheng, H-Y. Chen, and W. Dixon. Framework for guaranteed convergence of image-based observers with intermittent measurements. IEEE Transactions on Robotics, 33(2):266-280, 2017.

[16] E. Sontag. Mathematical Control Theory, Second Edition. Springer, New York, 1998. 